\title{
Antidepressants, antiplatelets and bleeding: One more thing to worry about?
}

\author{
David N. Juurlink MD PhD
}

See related research article by Labos and colleagues on page 1835 and at www.cmaj.ca/lookup/doi/10.1503/cmaj.100912

I n 2010, more than 25 million prescriptions for selective serotonin reuptake inhibitor (SSRI) antidepressants were dispensed in Canada (Josiane Gaudet, Brogan IMS, Kirkland, Quebec: personal communication, 2011). How many of these were dispensed to patients also taking acetylsalicylic acid (ASA), clopidogrel or both is not known, but most clinicians recognize that the concomitant use of antidepressants and antiplatelet drugs is common, particularly among elderly patients. But is the combination safe? In a related research article, Labos and colleagues used administrative data from Quebec to show that use of an SSRI was associated with a $42 \%$ increase in the relative risk of hemorrhage in patients taking ASA (v. ASA alone) and a $54 \%$ increase in the relative risk of hemorrhage in patients taking clopidogrel (v. clopidogrel alone). ${ }^{1}$ These findings will come as a surprise to many clinicians, but they add to a growing body of evidence that the use of SSRIs is an independent risk factor for hemorrhage.

Upon exposure to damaged endothelium, platelets adhere and become activated, releasing the cytokines contained in their granules. These molecules promote local vasoconstriction and further aggregation of platelets, creating a loose "plug" that stops bleeding. Thereafter, the activation of clotting factors culminates in the synthesis of fibrin, which meshes with the platelet plug to form a mature clot. Collectively, these processes constitute coagulation.

The targeted disruption of coagulation with drugs is an extremely powerful tool that, over the past 50 years, has saved millions of lives and averted untold morbidity. Overwhelming evidence shows that warfarin, which interrupts several vitamin $\mathrm{K}$-dependent steps in the coagulation cascade, markedly reduces the risk of embolic stroke in patients with atrial fibrillation. ${ }^{2}$ Newer, more targeted antithrombotic drugs such as dabigatran, rivaroxaban and apixaban do the same. But perhaps the best example is ASA, which acetylates (and therefore irreversibly inactivates) cyclooxygenase, the enzyme in platelets responsible for the synthesis of thromboxane A2, a compound that normally promotes activation and aggregation of platelets. Because platelets have no nuclei and cannot synthesize new cyclooxygenase, treatment with ASA renders them "less sticky" for the duration of their 7-10 day lifespan. This mechanism underlies the protective effects of ASA in patients with acute myocardial infarction and atherothrombotic stroke, conditions characterized by acute disruption of endothelial plaque that would otherwise prove irresistible to passing platelets. Also effective for these conditions are ticlopidine, clopidogrel and prasugrel, which inhibit platelet activation by blocking the pro-aggregatory effects of adenosine diphosphate.

Predictably, pharmacologic tinkering with platelets increases the risk of bleeding. In a meta-analysis of 50 randomized controlled trials, the overall rate of bleeding ranged from $3.6 \%$ at ASA doses of less than $100 \mathrm{mg} / \mathrm{d}$ to $8.5 \%$ during clopidogrel therapy; in most of these cases, bleeding was minor. ${ }^{3}$ Factors that increase the risk of hemorrhage during antiplatelet therapy include increasing age, a history of bleeding, dual antiplatelet therapy and particularly the concomitant use of oral anticoagulants. In a large retrospective study involving more than 80000 Danish patients with atrial fibrillation, those receiving ASA in combination with warfarin had a roughly twofold increase in the risk of hemorrhage relative to warfarin monotherapy, while those receiving "triple therapy" with ASA, clopidogrel and warfarin had a nearly fourfold increase in risk. ${ }^{4}$ The combined use of

\section{KEY POINTS}

- Antidepressants and antiplatelet agents are among the most widely prescribed medications in Canada.

- Converging lines of evidence suggest that SSRI antidepressants interfere with the aggregation of platelets and increase the risk of bleeding.

- The risks of therapy may outweigh the benefits for some patients taking antiplatelet medications and antidepressants, particularly those who experience a hemorrhage. 
antiplatelet agents with warfarin is justifiable for only a few patient groups, including those with mechanical heart valves and those with atrial fibrillation who experience acute myocardial infarction or who undergo stent placement following percutaneous coronary intervention. ${ }^{5}$ The commonly encountered scenario in which warfarin (or, for that matter, dabigatran or another of the newer antithrombotics) is prescribed for atrial fibrillation and ASA is prescribed for stable coronary artery disease should generally be viewed as a reason to discontinue ASA.

Why might SSRIs be associated with an increased risk of bleeding in patients taking antiplatelet drugs, as suggested by Labos and colleagues?' The answer lies in the mechanism of action of these drugs, which block the reuptake of serotonin in platelets as well as in neural tissue. As a result, the serotonin content of platelets is reduced, perhaps to as little as $1 \%$ of pretreatment values. ${ }^{6}$ Because serotonin plays an important role in the aggregation of platelets, SSRIs induce a qualitative platelet defect by a mechanism different from that of ASA or clopidogrel.

An association between SSRIs and hemorrhage has been described in case reports ${ }^{7}$ and some (but not all) observational studies. ${ }^{8.9}$ Some evidence suggests that the risk of hemorrhage correlates with the degree to which serotonin reuptake is inhibited. ${ }^{9,10}$ In one case-control study involving 64000 people newly prescribed antidepressants, the risk of admission to hospital because of bleeding was highest with fluoxetine, sertraline, clomipramine and paroxetine - drugs that exhibit a particularly high degree of inhibition of serotonin reuptake. ${ }^{10}$

The inhibitory effect of SSRIs on platelet activity is not necessarily bad news, however. In the SADHEART study, ${ }^{11} 369$ patients with major depression following discharge for acute coronary syndromes were randomly assigned to receive treatment with sertraline or placebo. Sertraline therapy was associated with a trend toward a lower risk of myocardial infarction, heart failure and death. Several observational studies have also suggested that, in patients with acute coronary syndromes, treatment with an SSRI is associated with improved cardiovascular outcomes. ${ }^{12,13}$

Based on converging lines of evidence, we can be confident that SSRIs alter platelet function and that some patients will bleed as a result. How should we use this information? The answer depends on the patient. The concomitant use of SSRIs and antiplatelet agents is not contraindicated and is sometimes essential. In general, the absolute risk is likely to be low. However, for many patients, it may be worth re-examining the overall goal of drug treatment, which is generally to offer a benefit that exceeds risk. Recognizing that SSRIs offer a meaningful advantage over placebo primarily in patients with severe depression $^{14}$ and that most patients who receive treatment have mild or moderate depressive symptoms, perhaps we should have a higher threshold to use these medications. For patients who experience a hemorrhage and have a strong indication for SSRI therapy, it may be worth preferentially avoiding drugs with a particularly high affinity for the serotonin receptor, including paroxetine, sertraline, fluoxetine and clomipramine.

\section{References}

1. Labos C, Dasgupta K, Nedjar H, et al. Risk of bleeding associated with combined use of selective serotonin reuptake inhibitors and antiplatelet therapy following acute myocardial infarction. CMAJ 2011;183:1835-43.

2. Lip GY, Edwards SJ. Stroke prevention with aspirin, warfarin and ximelagatran in patients with non-valvular atrial fibrillation: a systematic review and meta-analysis. Thromb Res 2006;118: 321-33.

3. Serebruany VL, Malinin AI, Eisert RM, et al. Risk of bleeding complications with antiplatelet agents: meta-analysis of 338,191 patients enrolled in 50 randomized controlled trials. Am J Hematol 2004;75:40-7.

4. Hansen ML, Sorensen R, Clausen MT, et al. Risk of bleeding with single, dual, or triple therapy with warfarin, aspirin, and clopidogrel in patients with atrial fibrillation. Arch Intern Med 2010;170:1433-41.

5. Douketis JD. Combination warfarin-ASA therapy: Which patients should receive it, which patients should not, and why? Thromb Res 2011;127:513-7.

6. Javors MA, Houston JP, Tekell JL, et al. Reduction of platelet serotonin content in depressed patients treated with either paroxetine or desipramine. Int J Neuropsychopharmacol 2000;3:229-35.

7. Lake MB, Birmaher B, Wassick S, et al. Bleeding and selective serotonin reuptake inhibitors in childhood and adolescence. J Child Adolesc Psychopharmacol 2000;10:35-8.

8. de Abajo FJ, Rodriguez LA, Montero D. Association between selective serotonin reuptake inhibitors and upper gastrointestinal bleeding: population based case-control study. BMJ 1999;319: 1106-9.

9. van Walraven C, Mamdani MM, Wells PS, et al. Inhibition of serotonin reuptake by antidepressants and upper gastrointestinal bleeding in elderly patients: retrospective cohort study. $B M J$ 2001;323:655-8.

10. Meijer WE, Heerdink ER, Nolen WA, et al. Association of risk of abnormal bleeding with degree of serotonin reuptake inhibition by antidepressants. Arch Intern Med 2004;164:2367-70.

11. Glassman AH, O'Connor CM, Califf RM, et al. Sertraline treatment of major depression in patients with acute MI or unstable angina. JAMA 2002;288:701-9.

12. Schlienger RG, Fischer LM, Jick H, et al. Current use of selective serotonin reuptake inhibitors and risk of acute myocardial infarction. Drug Saf 2004;27:1157-65.

13. Sauer WH, Berlin JA, Kimmel SE. Effect of antidepressants and their relative affinity for the serotonin transporter on the risk of myocardial infarction. Circulation. 2003 Jul 8;108:32-6. Epub 2003 Jun 23.

14. Fournier JC, DeRubeis RJ, Hollon SD, et al. Antidepressant drug effects and depression severity: a patient-level meta-analysis. JAMA 2010;303:47-53.

Affiliations: David Juurlink is with the Sunnybrook Research Institute, the Departments of Medicine, Pediatrics, and Health Policy, Management and Evaluation at the University of Toronto, and The Institute for Clinical Evaluative Sciences, Toronto, Ont.

Acknowledgements: The author thanks Moira Kapral and Victor Serebruany for comments on earlier versions of this commentary. 